Pacific Journal of Mathematics

WEAKLY COMPACT HOLOMORPHIC MAPPINGS ON 


\title{
WEAKLY COMPACT HOLOMORPHIC MAPPINGS ON BANACH SPACES
}

\author{
RAYMOND A. RYAN
}

\begin{abstract}
A holomorphic mapping $f: E \rightarrow F$ of complex Banach spaces is weakly compact if every $x \in E$ has a neighbourhood $V_{x}$ such that $f\left(V_{x}\right)$ is a relatively weakly compact subset of $F$. Several characterizations of weakly holomorphic mappings are given which are analogous to classical characterizations of weakly compact linear mappings and the DavisFigiel-Johnson-Pelczynski factorization theorem is extended to weakly compact holomorphic mappings. It is shown that the complex Banach space $E$ has the property that every holomorphic mapping from $E$ into an arbitrary Banach space is weakly compact if and only if the space $\mathscr{H}(E)$ of holomorphic complex-valued functions on $E$, endowed with the bornological topology $\tau_{\delta}$, is reflexive.
\end{abstract}

1. Introduction. Aron and Schottenloher [3], in a study of the approximation property for locally convex spaces of holomorphic functions on a complex Banach space, introduced the concept of a compact holomorphic mapping. If $E$ and $F$ are complex Banach spaces, a holomorphic mapping $f: E \rightarrow F$ is said to be compact if every $x \in E$ has a neighbourhood $V_{x}$ such that $f\left(V_{x}\right)$ is a relatively compact subset of $F$. They obtained several characterizations of compact holomorphic mappings which were analogous to characterizations of compact linear mappings. For example, it is well-known that a continuous linear mapping $T$ : $E \rightarrow F$ is compact if and only if its transpose $T^{t}: F^{\prime} \rightarrow E^{\prime}$ is compact. Now consider a holomorphic mapping $f: E \rightarrow F$. Denoting by $\mathscr{H}(E)$ the vector space of holomorphic functions on $E$ (that is, holomorphic mappings from $E$ into $\mathbf{C}$ ), the transpose of $f$ may be defined as the linear mapping $f^{t}: F^{\prime} \rightarrow \mathscr{H}(E)$ given by $f^{t}(\psi)=\psi \circ f$ for $\psi \in F^{\prime}$. Aron and Schottenloher show that when $\mathscr{H}(E)$ is given a suitable locally convex topology, the holomorphic mapping $f$ is compact if and only if its transpose $f^{t}$ is a compact linear mapping of $F^{\prime}$ into $\mathscr{H}(E)$.

Motivated by this work, we carry out a similar study of weakly compact holomorphic mappings, which are defined in the same way: a holomorphic mapping $f: E \rightarrow F$ is weakly compact if every $x \in E$ has a neighbourhood $V_{x}$ such that $f\left(V_{x}\right)$ is a relatively weakly compact subset of $F$. We extend some of the classical theory of weakly compact linear mappings to the holomorphic setting. Of course, some of our results are 
analogous to those of Aron and Schottenloher, but, just as in the linear case, the proofs for the compact and weakly compact situations are often quite different.

We begin in $\$ 2$ with weakly compact polynomials between Banach spaces. The properties of weakly compact polynomials which we require for the sequel follow easily from the fact that the continuous polynomials can be viewed as continuous linear mappings on a suitable tensor product.

In $\$ 3$ we consider weakly compact holomorphic mappings. We show that weak compactness of a holomorphic mapping is equivalent to weak compactness of its derivatives. Next, we show that weak compactness of a holomorphic mapping $f: E \rightarrow F$ is equivalent to weak compactness of its transpose $f^{t}: F^{\prime} \rightarrow\left(\mathscr{H}(E), \tau_{\omega}\right)$ where $\tau_{\omega}$ is the Nachbin topology of $\mathscr{H}(E)$, and that this is in turn equivalent to $f^{t t}\left(\left(\mathscr{H}(E), \tau_{\omega}\right)^{\prime}\right) \subset F$, generalising the fact that a continuous linear mapping $T: E \rightarrow F$ is weakly compact if and only if $T^{t t}\left(E^{\prime \prime}\right) \subset F$. This, and other results in this paper, reinforce the view expressed in [3] that $\mathscr{H}(E)$ can be thought of as a "holomorphic dual" of $E$. Next, we show that the Davis-FigielJohnson-Pekczynski theorem, which states that weakly compact linear mappings factor through reflexive spaces, extends to holomorphic mappings. Finally, we examine the relationship between weakly compact holomorphic mappings and reflexivity. We show that $E$ has the property that for every compact Banach space $F$, every holomorphic mapping from $E$ into $F$ is weakly compact, if and only if the locally convex space $\left(\mathscr{H}(E), \tau_{\delta}\right)$ is reflexive, where $\tau_{\delta}$ is the bornological topology associated with $\tau_{\omega}$.

We now outline our notation and terminology. $E$ and $F$ denote complex Banach spaces. $E^{\prime}, F^{\prime}$ denote their strong duals, and $F_{\tau}^{\prime}$ denotes the dual space of $F$ endowed with the Mackey topology $\tau\left(F^{\prime}, F\right) . \bar{\Gamma} U$ denotes the closed convex balanced hull of the set $U$. If $X$ is a locally convex space, $X_{\beta}^{\prime}$ denotes the strong dual of $X$, that is the dual space of $X$ with the topology of uniform convergence on bounded subsets of $X$. For $n \in \mathbf{N}, L_{s}\left({ }^{n} E ; F\right)$ denotes the Banach space of continuous symmetric $n$-linear mappings from $E$ into $F$, where the norm is given by $\|A\|=$ $\sup \left\{\left\|A\left(x_{1}, \ldots, x_{n}\right)\right\|:\left\|x_{1}\right\|, \ldots,\left\|x_{n}\right\| \leq 1\right\}$. A mapping $P: E \rightarrow F$ is a continuous $n$-homogeneous polynomial if there exists a necessarily unique element $A$ of $L_{s}\left({ }^{n} E ; F\right)$ such that $P(x)=A(x, \ldots, x)$ for every $x \in E$. $P\left({ }^{n} E ; F\right)$ denotes the Banach space of continuous $n$-homogeneous polynomials from $E$ into $F$ with the norm given by $\|P\|=\sup \{\|P(x)\|:\|x\|$ $\leq 1\}$. The mapping $P \rightarrow A$ is an isomorphism from $P\left({ }^{n} E ; F\right)$ onto $L_{s}\left({ }^{n} E ; F\right)$. 
A mapping $f: E \rightarrow F$ is holomorphic if for each $x \in E$ there exists a sequence of polynomials

$$
\frac{1}{n !} \hat{d}^{n} f(x) \in P\left({ }^{n} E ; F\right)
$$

and a neighbourhood $V$ of $x$ such that the series

$$
\sum_{n=0}^{\infty} \frac{1}{n !} \hat{d}^{n} f(x)(y-x)
$$

converges uniformly to $f(y)$ for $y \in V$. $\mathscr{H}(E ; F)$ denotes the vector space of holomorphic mappings from $E$ into $F$. When $F=\mathbf{C}$ the spaces of functions which we have introduced above are denoted $L_{s}\left({ }^{n} E\right), P\left({ }^{n} E\right)$ and $\mathscr{H}(E)$. A seminorm $p$ on $\mathscr{H}(E ; F)$ is said to be ported by a compact subset $K$ of $E$ if for every neighbourhood $V$ of $K$ there exists $C_{V}>0$ such that $p(f) \leq C_{V}\|f\|_{V}$ for every $f \in \mathscr{H}(E ; F)$. The Nachbin topology, $\tau_{\omega}$ is the locally convex topology on $\mathscr{H}(E ; F)$ generated by the seminorms which are ported by compact subsets of $E . \tau_{\omega}$ induces the norm topology on the subspaces $P\left({ }^{n} E ; F\right)$ of $\mathscr{H}(E ; F)$. We refer to [6] and [11] for further details about polynomials and holomorphic mappings.

Most of the results given here are from the author's Ph. D. dissertation, which was written under the supervision of Dr. R. M. Aron. The rest of the research for this paper was carried out while the author was visiting Kent State University, to which thanks are acknowledged.

2. Weakly compact polynomials. Let $P$ be a continuous $n$-homogeneous polynomial from $E$ into $F$ and let $A_{P}$ be the unique continuous symmetric $n$-linear mapping from $E^{n}$ into $F$ which generates $P$, so that $P(x)=A_{P}(x, \ldots, x)$ for every $x \in E$. Let $L_{P} \in L\left(\hat{\otimes}^{n} E ; F\right)$ be the continuous linear mapping associated with $A_{P}$ where $\hat{\otimes}^{n} E=E \hat{\otimes} \cdots \hat{\otimes} E$ is the $n$-fold projective tensor product [8]. The polynomial $P$ is said to be weakly compact if $P$ maps the unit ball of $E$ into a relatively weakly compact subset of $F$. It is shown in [12] that $P$ is weakly compact if and only if the associated linear mapping $L_{P}$ is weakly compact.

The transpose of $P \in P\left({ }^{n} E ; F\right)$ is the continuous linear mapping $P^{t}$. $F^{\prime} \rightarrow P\left({ }^{n} E\right)$ defined by $P^{t}(\psi)=\psi \circ P$ for $\psi \in F^{\prime}$. We wish to relate weak compactness of $P$ to the behaviour of the linear mapping $P^{t} . P^{t}$ can be identified with the transpose of the linear mapping $L_{P}$ in the following way: $L_{P}^{t}$ maps $F^{\prime}$ into $L_{s}\left({ }^{n} E\right)$, and $J \circ P^{t}=L_{P}^{t}$, where $J: P\left({ }^{n} E\right) \rightarrow$ $L_{s}\left({ }^{n} E\right)$ is the isomorphism given by $J(Q)=A_{Q}$. We now apply the following well-known equivalent formulations of weak compactness of linear mappings (see, for example [7]). The following properties of a 
continuous linear mapping $T: X \rightarrow Y$ are equivalent:

(a) $T$ is weakly compact;

(b) $T^{t}: Y_{\tau}^{\prime} \rightarrow X^{\prime}$ is continuous;

(c) $T^{t}: Y^{\prime} \rightarrow X^{\prime}$ is weakly compact.

Applying this to the linear mapping $L_{P}$, we obtain:

Proposition 2.1. Let $P \in P\left({ }^{n} E ; F\right)$. The following are equivalent:

(i) $P$ is weakly compact;

(ii) $P^{t}: F_{\tau}^{\prime} \rightarrow P\left({ }^{n} E\right)$ is continuous;

(iii) $P^{t}: F^{\prime} \rightarrow P\left({ }^{n} E\right)$ is weakly compact.

$P_{w K}\left({ }^{n} E ; F\right)$ denotes the subspace of $P\left({ }^{n} E ; F\right)$ of weakly compact $n$-homogeneous polynomials from $E$ into $F$.

Corollary 2.2. $P_{w K}\left({ }^{n} E ; F\right)$ is a closed subspace of $P\left({ }^{n} E ; F\right)$.

Proof. Let $\left\{P_{n}\right\}$ be a sequence in $P_{w K}\left({ }^{n} E ; F\right)$ which converges to $P \in P\left({ }^{n} E ; F\right)$. Then $P_{n}^{t}$ converges to $P^{t}$ in $L\left(F^{\prime} ; P\left({ }^{n} E\right)\right)$ and since $L_{w K}\left(F^{\prime} ; P\left({ }^{n}(E)\right)\right.$ is closed in $L\left(F^{\prime} ; P\left({ }^{n} E\right)\right)$, it follows that $P^{t}$, and hence $P$, is weakly compact.

For further results concerning weakly compact polynomials on Banach spaces with the Dunford-Pettis property, we refer to $[12,13]$.

3. Weakly compact holomorphic mappings. We shall say that a holomorphic mapping $f: E \rightarrow F$ is weakly compact if every $x \in E$ has a neighbourhood $V_{x}$ such that $f\left(V_{x}\right)$ is a relatively weakly compact subset of $F$. Our first objective is to establish a connection between weak compactness of a holomorphic mapping and weak compactness of its derivatives.

We call a subset $U$ of $E$ circled if for every $x \in U$ and every $\lambda \in \mathbf{C}$ with $|\lambda|=1$, we have $\lambda x \in U$. The following lemma is an easy application of the Hahn-Banach Theorem and the Cauchy inequality (see the proof of [3, Proposition 3.4] for a similar result).

LEMMA 3.1. Let $f \in \mathscr{H}(E ; F)$. If $U \subset E$ is circled and $x \in E$, then

$$
\frac{1}{n !} \hat{d}^{n} f(x)(U) \subset \bar{\Gamma} f(x+U)
$$

for every $n$.

We now show that weak compactness of a holomorphic mapping $f$ is equivalent to weak compactness of its derivatives at every point, and also that weak compactness of $f$ is determined by the behaviour of $f$ at the 
origin. We refer to [3, Proposition 3.4] for an analogous result for compact holomorphic mappings.

THEOREM 3.2. Let $f \in \mathscr{H}(E ; F)$. The following are equivalent:

(i) $f$ is weakly compact;

(ii) $f$ maps some neighbourhood of the origin in $E$ into a relatively weakly compact subset of $F$;

(iii) $\hat{d}^{n} f(x)$ is a weakly compact polynomial for every $n \in \mathbf{N}$ and every $x \in E$;

(iv) $\hat{d}^{n} f(0)$ is a weakly compact polynomial for every $n \in \mathbf{N}$.

Proof. The implications (iii) $\Rightarrow$ (iv) and (i) $\Rightarrow$ (ii) are trivial, while (i) $\Rightarrow$ (iii) and (ii) $\Rightarrow$ (iv) follow immediately from Lemma 3.1. It only remains to show that (iv) implies (i).

Suppose, therefore, that $\hat{d}^{n} f(0)$ is a weakly compact polynomial for every $n \in \mathbf{N}$. Let $X=\left\{x \in E: f\left(V_{x}\right)\right.$ is relatively weakly compact for some neighbourhood $V_{x}$ of $x$ \}. We shall prove that $X$ is a non-empty subset of $E$ and is both open and closed, from which it follows that $f$ is weakly compact.

First we show that $X$ contains the origin. Choose $r>0$ so that $f$ is bounded on the ball $B(0, r)$. We claim that $f(B(0, r / 2))$ is a relatively weakly compact subset of $F$. To see this, let $K_{n}=\bar{\Gamma}\left((1 / n !) \hat{d}^{n} f(0)(B(0, r))\right)$. Since $(1 / n !) \hat{d}^{n} f(0)$ is a weakly compact polynomial, $K_{n}$ is weakly compact for every $n$. By Lemma $3.1 K_{n}$ is contained in the bounded set $\bar{\Gamma} f(B(0, r))$ for every $n$, and therefore if $z_{n} \in K_{n}$ then the series $\sum_{n=0}^{\infty} 2^{-n} z_{n}$ converges absolutely. Therefore $K=\left\{\sum_{n=0}^{\infty} 2^{-n} z_{n}: z_{n} \in K_{n}\right\}$ is a well-defined bounded subset of $F$. Now, for $x \in B(0, r / 2)$, we have

$$
f(x)=\sum_{n=0}^{\infty} \frac{1}{n !} \hat{d}^{n} f(0)(x)=\sum_{n=0}^{\infty} 2^{-n}\left(\frac{1}{n !} \hat{d}^{n} f(0)(2 x)\right),
$$

and hence $f(B(0, r / 2)) \subset K$. We show that $K$ is weakly compact by proving that for every $\psi \in F^{\prime}$ there exists $z \in K$ such that $\psi(z)=\|\psi\|_{K}$ [10].

Let $\psi \in F^{\prime}$. Since $K_{n}$ is weakly compact and balanced, there exist $w_{n} \in K_{n}$ such that $\psi\left(w_{n}\right)=\|\psi\|_{K_{n}}$. Then $w=\sum_{n=0}^{\infty} 2^{-n} w_{n} \in K$, and

$$
\|\psi\|_{K} \leq \sum_{n=0}^{\infty} 2^{-n}\|\psi\|_{K_{n}}=\sum_{n=0}^{\infty} 2^{-n} \psi\left(w_{n}\right)=\psi(w) \leq\|\psi\|_{K} .
$$

Therefore $\psi(w)=\|\psi\|_{K}$ and our claim is proved.

It is clear that $X$ is an open subset of $F$. Let $\left\{x_{k}\right\}$ be a sequence in $X$, converging to $x \in E$. By Lemma $3.1 \hat{d}^{n} f\left(x_{k}\right)$ is weakly compact for every $n$ and every $k$. Since $P_{w K}\left({ }^{n} E ; F\right)$ is a closed subspace of $P\left({ }^{n} E ; F\right)$, and 
$\lim _{k \rightarrow \infty} \hat{d}^{n} f\left(x_{k}\right)=\hat{d}^{n} f(x)$ in $P\left({ }^{n} E ; F\right)$, it follows that $\hat{d}^{n} f(x)$ is weakly compact for every $n$. Consider the holomorphic mapping $g: E \rightarrow F$ defined by $g(z)=f(x+z)$. We have $\hat{d}^{n} g(0)=\hat{d}^{n} f(x)$ for every $n$, and it follows from the first part of this proof that $g$ maps some neighbourhood $V$ of the origin into a relatively weakly compact subset of $F$. Therefore $f$ maps the neighbourhood $x+V$ of $x$ into a relatively weakly compact set, which shows that $x \in X$. Therefore $X$ is closed.

We shall denote by $\mathscr{H}_{w K}(E ; F)$ the vector space of weakly compact holomorphic mappings from $E$ into $F$.

Corollary 3.3. $\left(\mathscr{H}_{w K}(E ; F), \tau_{\omega}\right)$ is a closed subspace of $\left(\mathscr{H}(E ; F), \tau_{\omega}\right)$.

Proof. Let $\left\{f_{\lambda}\right\}$ be a net in $\mathscr{H}_{w K}(E ; F)$ which converges in the topology $\tau_{\omega}$ to $f \in \mathscr{H}(E ; F)$. Since $\tau_{\omega}$ induces the norm topology on $P\left({ }^{n} E ; F\right)$ it follows that $\hat{d}^{n} f_{\lambda}(0)$ converges to $\hat{d}^{n} f(0)$ in the norm of $P\left({ }^{n} E ; F\right)$ for every $n$. But $\hat{d}^{n} f_{\lambda}(0) \in P_{w K}\left({ }^{n} E ; F\right)$ for every $\lambda$, and $P_{w K}\left({ }^{n} E ; F\right)$ is closed in $P\left({ }^{n} E ; F\right)$. Therefore, by Theorem 3.2, $f$ is weakly compact.

If $f$ is a holomorphic mapping from $E$ to $F$ then, following Aron and Schottenloher, we define the transpose of $f$ to the linear mapping $f^{t}$ : $F^{\prime} \rightarrow \mathscr{H}(E)$ given by $f^{t}(\psi)=\psi \circ f$. This mapping is continuous for the norm topology on $F^{\prime}$ and the Nachbin topology $\tau_{\omega}$ on $\mathscr{H}(E)$. To see this, let $p$ be a $\tau_{\omega}$-continuous seminorm on $\mathscr{H}(E)$, so that $p$ is ported by some compact subset $K$ of $E$. Thus, for every open neighbourhood $V$ of $K$ there exists $C_{V}>0$ such that $p(g) \leq C_{V}\|g\|_{V}$ for every $g \in \mathscr{H}(E)$. Choose an open neighbourhood $V$ of $K$ for which $f(V)$ is bounded. Then $p\left(f^{t}(\psi)\right) \leq C_{V}\left\|f^{t}(\psi)\right\|_{V}=C_{V}\|\psi\|_{f(V)}$, and hence $p \circ f^{t}$ is a continuous seminorm on $F^{\prime}$.

Since $f^{t}: F^{\prime} \rightarrow\left(\mathscr{H}(E), \tau_{\omega}\right)$ is a continuous linear mapping, we may take its transpose in the usual linear sense to obtain a linear mapping $f^{t t}$ : $\left(\mathscr{H}(E), \tau_{\omega}\right)^{\prime} \rightarrow F^{\prime \prime}$. Our next result shows that the transpose and bitranspose of a holomorphic mapping can be used to characterize weak compactness. We recall that for a continuous linear mapping $T: E \rightarrow F$, weak compactness of $T$ is equivalent to each of the following conditions:

(a) $T^{t}: F^{\prime} \rightarrow E^{\prime}$ is weakly compact;

(b) $T^{t t}$ maps $E^{\prime \prime}$ into the canonical image of $F$ in $F^{\prime \prime}$. 
For holomorphic mappings we have: (cf. [3, Proposition 3.6])

THEOREM 3.4. Let $f \in \mathscr{H}(E ; F)$. The following are equivalent:

(i) $f$ is weakly compact;

(ii) $f^{t}: F_{\tau}^{\prime} \rightarrow\left(\mathscr{H}(E), \tau_{\omega}\right)$ is continuous, where $\tau=\tau\left(F^{\prime}, F\right)$ is the Mackey topology of $F^{\prime}$;

(iii) $f^{t}: F^{\prime} \rightarrow\left(\mathscr{H}(E), \tau_{\omega}\right)$ is weakly compact;

(iv) $f^{t t}$ maps $\left(\mathscr{H}(E), \tau_{\omega}\right)^{\prime}$ into the canonical image of $F$ in $F^{\prime \prime}$.

Proof. (i) implies (ii): Let $p$ be a continuous seminorm on $\left(\mathscr{H}(E), \tau_{\omega}\right)$. Then $p$ is ported by some compact subset $K$ of $E$, and so for every open set $U$ containing $K$ there exists $C_{U}>0$ such that $p(g) \leq C_{U}\|g\|_{U}$ for every $g \in \mathscr{H}(E)$. Since $f$ is weakly compact there exists an open neighbourhood $V$ of $K$ such that $f(V)$ is a relatively weakly compact subset of $F$. Therefore $\psi \rightarrow\|\psi\|_{f(V)}$ is a continuous seminorm on $F_{\tau}^{\prime}$, and since

$$
p\left(f^{t}(\psi)\right) \leq C_{V}\left\|f^{t}(\psi)\right\|_{V}=C_{V}\|\psi\|_{f(V)},
$$

it follows that $f^{t}: F_{\tau}^{\prime} \rightarrow\left(\mathscr{H}(E), \tau_{\omega}\right)$ is continuous.

(ii) implies (iii): It follows from (ii) that $f^{t}$ is continuous for the weak topologies $\sigma\left(F^{\prime}, F\right)$ and $\sigma\left(\mathscr{H}(E),\left(\mathscr{H}(E), \tau_{\omega}\right)^{\prime}\right)$. Since the closed unit ball of $F^{\prime}$ is $\sigma\left(F^{\prime}, F\right)$-compact, it is mapped by $f^{t}$ into a weakly compact subset of $\left(\mathscr{H}(E), \tau_{\omega}\right)$.

(iii) implies (i): The mapping $g \rightarrow \hat{d}^{n} g(0)$ is a continuous projection of $\left(\mathscr{H}(E), \tau_{\omega}\right)$ onto the subspace $P\left({ }^{n} E\right)$. If we compose $f^{t}$ with this mapping we obtain the continuous mapping $\left(\hat{d}^{n} f(0)\right)^{t}: F_{\tau}^{\prime} \rightarrow P\left({ }^{n} E\right)$. It follows from Proposition 2.1 that $\hat{d}^{n} f(0)$ is a weakly compact polynomial for every $n$ and hence, by Theorem 3.2, $f$ is weakly compact.

(ii) implies (iv) is trivial, since $\left(F_{\tau}^{\prime}\right)^{\prime}$ is the canonical image of $F$ in $F^{\prime \prime}$. To see that (iv) implies (ii), suppose that $f^{t t}$ maps $\left(\mathscr{H}(E), \tau_{\omega}\right)^{\prime}$ into the canonical image of $F$ in $F^{\prime \prime}$. Then $f^{t}$ is continuous for the weak topologies $\sigma\left(F^{\prime}, F\right)$ and $\sigma\left(\mathscr{H}(E),\left(\mathscr{H}(E), \tau_{\omega}\right)^{\prime}\right)$, and it follows that $f^{t}$ is continuous for the Mackey topologies associated with these weak topologies. But $\tau_{\omega}$ is weaker than the Mackey topology associated with it, and hence $f^{t}$ is a continuous mapping of $F_{\tau}^{\prime}$ into $\left(\mathscr{H}(E), \tau_{\omega}\right)$.

Now by Theorem 3.4 if $f \in \mathscr{H}_{w K}(E ; F)$ then the transpose $f^{t}$ belongs to the space $L\left(F_{\tau}^{\prime} ;\left(\mathscr{H}(E), \tau_{\omega}\right)\right)$. We shall endow this space of continuous linear mappings with the topology of uniform convergence on the equicontinuous subset of $F^{\prime}$, and we denote the resulting locally convex space by $L_{\varepsilon}\left(F_{\tau}^{\prime} ;\left(\mathscr{H}(E), \tau_{\omega}\right)\right)$. We wish to show that the mapping $f \rightarrow f^{t}$ establishes an isomorphism of $\left(\mathscr{H}_{w K}(E ; F), \tau_{\omega}\right)$ with $L_{\varepsilon}\left(F_{\tau}^{\prime} ;\left(\mathscr{H}(E), \tau_{\omega}\right)\right)$. 
For each $x \in E$ let $\delta(x)$ be the continuous linear functional on $\left(\mathscr{H}(E), \tau_{\omega}\right)$ given by $f \rightarrow f(x)$.

LEMMA 3.5. $\delta$ is a holomorphic mapping from $E$ into $\left(\mathscr{H}(E), \tau_{\omega}\right)_{\beta}^{\prime}$.

Proof. We show that $\delta$ has a Fréchet derivative at every point of $E$. For each $x \in E$ let $\pi_{x}$ denote the continuous projection of $\left(\mathscr{H}(E), \tau_{\omega}\right)$ onto the subspace $E^{\prime}$ which associates with $f \in \mathscr{H}(E)$ its derivative at $x$, $d f(x)$. Let $J$ denote the canonical embedding of $E$ into $E^{\prime \prime}$. We claim that the continuous linear mapping $\pi_{x}^{t} \circ J$ is the Fréchet derivative of $\delta$ at $x$. Thus, we must show that

$$
\lim _{\|y\| \rightarrow 0} \frac{p\left[\delta(x+y)-\delta(x)-\pi_{x}^{t} \circ J(y)\right]}{\|y\|}=0
$$

for every continuous seminorm $p$ on $\left(\mathscr{H}(E), \tau_{\omega}\right)_{\beta}^{\prime}$. By the definition of the strong topology, we may assume without loss of generality that there exists a bounded subset $A$ of $\left(\mathscr{H}(E), \tau_{\omega}\right)$ such that $p(\phi)=\sup \{|\phi(f)|$ : $f \in A\}$ for every $\phi \in\left(\mathscr{H}(E), \tau_{\omega}\right)^{\prime}$. By [11, $\$ 12$, Proposition 1] there exist positive real numbers $C, \gamma$ such that $\left\|(1 / n !) \hat{d}^{n} f(x)\right\| \leq C \gamma^{n}$ for every $f \in A$. Therefore, if $\|y\|<\gamma^{-1}$ we have

$$
\begin{aligned}
& \frac{p\left[\delta(x+y)-\delta(x)-\pi_{x}^{t} \circ J(y)\right]}{\|y\|} \\
& =\|y\|^{-1} \sup _{f \in A}|f(x+y)-f(x)-d f(x)(y)| \\
& =\|y\|^{-1} \sup _{f \in A}\left|\sum_{n=2}^{\infty} \frac{1}{n !} \hat{d}^{n} f(x)(y)\right| \leq\|y\|^{-1} \sum_{n=2}^{\infty} C \gamma^{n}\|y\|^{n}=\frac{C \gamma^{2}\|y\|}{1-\gamma\|y\|},
\end{aligned}
$$

and since this tends to zero with $\|y\|$, our claim is proved.

Aron and Schottenloher show that the mapping $f \rightarrow f^{t}$ establishes an isomorphism between the space $\left(\mathscr{H}_{K}(E ; F), \tau_{\omega}\right)$ of compact holomorphic mappings from $E$ into $F$ endowed with the Nachbin topology, and the space $L_{\varepsilon}\left(F_{c}^{\prime} ;\left(\mathscr{H}(E), \tau_{\omega}\right)\right)$, where $F_{c}^{\prime}$ is the dual space of $F$ with the topology of uniform convergence on compact subset of $F[3$, Theorem 4.1] and $\varepsilon$ denotes the topology of uniform convergence on equicontinuous sets. In the weakly compact case we have an analogous result:

Proposition 3.6. The mapping $f \rightarrow f^{t}$ is an isomorphism from $\left(\mathscr{H}_{w K}(E ; F), \tau_{\omega}\right)$ onto $L_{\varepsilon}\left(F_{\tau}^{\prime} ;\left(\mathscr{H}(E), \tau_{\omega}\right)\right)$. 
Proof. It is easy to see that the mapping $f \rightarrow f^{t}$ is linear and injective. To see that this mapping is surjective, let $T \in L\left(F_{\tau}{ }^{\prime} ;\left(\mathscr{H}(E), \tau_{\omega}\right)\right)$. Then $T^{t} \in L\left(\left(\mathscr{H}(E), \tau_{\omega}\right)_{\tau}^{\prime} ; F\right)$ and therefore by Lemma $3.5 f=T^{t} \circ \delta$ is a holomorphic mapping from $E$ into $F$. If $\psi \in F^{\prime}$ and $x \in E$ then

$$
\left(f^{t} \psi\right)(x)=\langle\psi, f(x)\rangle=\left\langle\psi, T^{t} \delta(x)\right\rangle=\langle T \psi, \delta(x)\rangle=(T \psi)(x)
$$

Therefore $f^{t}=T$ and it follows that the mapping $f \rightarrow f^{t}$ is a bijection from $\mathscr{H}_{w K}(E ; F)$ onto $L\left(F_{\tau}^{\prime} ;\left(\mathscr{H}(E), \tau_{\omega}\right)\right)$ with inverse given by $T \rightarrow T^{t} \circ \delta$.

The proof that the mapping $f \rightarrow f^{t}$ and its inverse are continuous is the same as the proof of the analogous assertion for the compact case [3, Theorem 4.1].

Davis, Figiel, Johnson and Pelczynski [4] have shown that weakly compact linear mappings factor through reflexive spaces in the following sense: if $T$ is a weakly compact linear mapping of $E$ into $F$, there exists a reflexive Banach space $G$ and continuous linear mappings $S: E \rightarrow G, R$ : $G \rightarrow F$ such that $T=R \circ S$. We show next that weakly compact holomorphic mappings also factor through reflexive spaces.

If $A$ is a closed, convex, balanced and bounded subset of a Banach space $F$, then $F_{A}$ will denote the Banach space obtained by taking the linear span of $A$ with norm given by the Minkowski functional of $A$, so that $A$ is the closed unit ball of $F_{A}$.

Aron and Schottenloher show that compact holomorphic mappings have "thin" images, in the following sense: $f \in \mathscr{H}(E ; F)$ is compact if and only if there exists a compact, convex, balanced subset $K$ of $F$ such that $f(x) \in F_{K}$ for every $x \in E$, and $f$ is holomorphic as a mapping from $E$ into $F_{K}$ [3, Proposition 3.5]. We prove a similar result for weakly compact holomorphic mappings, and the Davis-Figiel-Johnson-Pelczynski theorem then enables us to factor these mappings through reflexive spaces.

THEOREM 3.7. Let $f \in \mathscr{H}(E ; F)$. The following are equivalent:

(i) $f$ is weakly compact;

(ii) There exists a weakly compact convex balanced subset $K$ of $F$ such that $f$ is a holomorphic mapping from $E$ into $F_{K}$;

(iii) There exists a reflexive Banach space $G$, a continuous linear mapping $T: G \rightarrow F$ and a holomorphic mapping $g: E \rightarrow F$ such that $f=T \circ g$. 
Proof. (i) implies (ii): As in the proof of [3, Proposition 3.5], there exists a sequence $\left\{U_{m}\right\}$ of open circled subsets of $E$ such that $f\left(U_{m}\right)$ is a bounded subset of $F$ for every $m$, and $E=\bigcup_{m=1}^{\infty} U_{m}$. Let $W_{m}$ be the balanced hull of $U_{m}$. Since $U_{m}$ is circled, it follows from the maximum modulus theorem that $f\left(W_{m}\right)$ is bounded for every $m$. Let $V_{m}=\frac{1}{2} W_{m}$. Then $E=\bigcup_{m=1}^{\infty} V_{m}$ and hence $f(E)=\bigcup_{m=1}^{\infty} f\left(V_{m}\right)$. Let

$$
K_{m n}=\bar{\Gamma}\left(\frac{1}{n !} \hat{d}^{n} f(0)\left(W_{m}\right)\right) .
$$

Then $K_{m n}$ is a weakly compact convex balanced subset of $F$ and by Lemma 3.1 $K_{m n} \subset \bar{\Gamma} f\left(W_{m}\right)$ for every $m, n$. Therefore, arguing in the same way as in the proof of Theorem 3.2, we find that the set

$$
K_{m}=\left\{\sum_{n=0}^{\infty} 2^{-n} z_{n}: z_{n} \in K_{m n}\right\}
$$

is weakly compact, and that $f\left(V_{m}\right) \subset K_{m}$ for every $m$. Hence $\bar{\Gamma} f\left(V_{m}\right)$ is a weakly compact convex balanced subset of $F$ for every $m$, and

$$
f(E) \subset \bigcup_{m=1}^{\infty} \bar{\Gamma} f\left(V_{m}\right) .
$$

Since $\bar{\Gamma} f\left(V_{m}\right)$ is a weakly compact convex balanced set for every $m$, there exists a sequence $\left\{\beta_{m}\right\}$ of positive real numbers such that $K=$ $\bigcup_{m=1}^{\infty} \beta_{m} \bar{\Gamma} f\left(V_{m}\right)$ is weakly compact [9, page 184]. It follows from (1) that $f$ maps $E$ into $F_{K}$. That $f$ is a holomorphic mapping from $E$ into $F_{K}$ is proved in exactly the same way as the analogous result for compact holomorphic mappings [3, Proposition 3.5].

(ii) implies (iii): Suppose there exists a weakly compact convex balanced subset $K$ of $F$ such that $f$ is a holomorphic mapping from $E$ into $F_{K}$. Let $f_{K}: E \rightarrow F_{K}$ be the mapping $x \rightarrow f(x)$ and let $J$ denote the injection of $F_{K}$ into $F$, so that $f=J \circ f_{K}$. Applying the Davis-FigielJohnson-Pekczynski theorem to $J$, there exists a reflexive Banach space $G$, and continuous linear mappings $T: G \rightarrow F$ and $S: F_{K} \rightarrow G$ such that $J=T \circ S$. Let $g=S \circ f_{K}$. Then $f=J \circ f_{K}=T \circ S \circ f_{K}=T \circ g$.

(iii) implies (i) is trivial.

We now look at the connection between reflexivity and weakly compact holomorphic mappings. It is easy to see that the Banach space $E$ is reflexive if and only if for every Banach space $F$, every continuous linear mapping from $E$ into $F$ is weakly compact. We wish to characterize those Banach spaces $E$ with the property that every holomorphic mapping with domain $E$ is weakly compact. 
The bornological topology on $\mathscr{H}(E)$ associated with the Nachbin topology $\tau_{\omega}$ is denoted by $\tau_{\delta}$. This topology is also the barreled topology associated with $\tau_{\omega}$ [6]. Dineen has shown that $\left(\mathscr{H}(E), \tau_{\delta}\right)$ is reflexive if and only if $P\left({ }^{n} E\right)$ is a reflexive Banach space for every $n$ [5, Proposition $2.5]$.

THEOREM 3.8. Let E be a complex Banach space. The following are equivalent:

(i) $\left(\mathscr{H}(E), \tau_{\delta}\right)$ is reflexive;

(ii) for every complex Banach space $F$ every holomorphic mapping from $E$ into $F$ is weakly compact.

Proof. (i) implies (ii): Let $f \in \mathscr{H}(E ; F)$. Consider the continuous linear mapping $f^{t}: F^{\prime} \rightarrow\left(\mathscr{H}(E), \tau_{\omega}\right)$. Since $\tau_{\delta}$ and $\tau_{\omega}$ have the same bounded sets, $f^{t}$ is a continuous linear mapping of $F^{\prime}$ into $\left(\mathscr{H}(E), \tau_{\delta}\right)$. Since $\left(\mathscr{H}(E), \tau_{\delta}\right)$ is reflexive, $f^{t}$ maps the unit ball of $F^{\prime}$ into a relatively weakly compact subset of $\left(\mathscr{H}(E), \tau_{\delta}\right)$. But $\tau_{\delta}$ is finer than $\tau_{\omega}$ and so $f^{t}$ is a weakly compact linear mapping from $F^{\prime}$ into $\left(\mathscr{H}(E), \tau_{\omega}\right)$. Hence by Theorem $3.4 f$ is weakly compact.

(ii) implies (i): For each $n$ let $\delta_{n}: E \rightarrow P\left({ }^{n} E\right)^{\prime}$ be the continuous $n$-homogeneous polynomial defined by $\left\langle\delta_{n}(x), P\right\rangle=P(x)$ for $P \in P\left({ }^{n} E\right)$. By (ii) $\delta_{n}$ is weakly compact and therefore by Proposition $2.1 \delta_{n}^{t}$ is a weakly compact linear mapping from $P\left({ }^{n} E\right)^{\prime \prime}$ into $P\left({ }^{n} E\right)$. But the restriction of $\delta_{n}^{t}$ to $P\left({ }^{n} E\right)$ is the identity mapping on $P\left({ }^{n} E\right)$. Therefore $P\left({ }^{n} E\right)$ is reflexive for every $n$.

Now $E^{\prime}$ is a subspace of $\left(\mathscr{H}(E), \tau_{\delta}\right)$ and so if $\left(\mathscr{H}(E), \tau_{\delta}\right)$ is reflexive then in particular $E$ is reflexive. The converse, however, is false. For example, $\left(\mathscr{H}\left(l_{p}\right), \tau_{\delta}\right)$ is not reflexive for any $p$ [1]. Examples of spaces $E$ for which $\left(\mathscr{H}(E), \tau_{\delta}\right)$ is reflexive are Tsirelson's space $T^{\prime}[\mathbf{1}]$, and all quotient spaces of $T^{\prime}[2]$.

\section{REFERENCES}

[1] R. Alencar, R. M. Aron and S. Dineen, A reflexive space of holomorphic functions in infinitely many variables, Proc. Amer. Math. Soc., 90 (1984), 407-411.

[2] R. M. Aron, L. A. Moraes and R. A. Ryan, Factorization of holomorphic mappings in infinite dimension, preprint.

[3] R. M. Aron and M. Schottenloher, Compact holomorphic mappings on Banach spaces and the approximation property, J. Funct. Anal., 21 (1976), 7-30.

[4] W. J. Davis, T. Figiel, W. B. Johnson and A. Pelczynski, Factoring weakly compact operators, J. Funct. Anal., 17 (1974), 311-327. 
[5] S. Dineen, Holomorphic functions on locally convex topological vector spaces: I Locally convex topologies on $\mathscr{H}(U)$, Ann. Inst. Fourier, Grenoble, 23 (1973), 19-54.

[6] , Complex Analysis in Locally Convex Spaces, Math. Studies 57, North-Holland, Amsterdam, 1981.

[7] R. E. Edwards, Functional Analysis, Theory and Applications, Holt Rinehart and Winston, New York, 1965.

[8] A. Grothendieck, Produits Tensoriels Topologiques et Espaces Nucleaires, Mem. Amer. Math. Soc. 16, American Mathematical Society, Providence, R. I., 1966.

[9] _ Topological Vector Spaces, Gordon and Breach, New York, 1973.

[10] R. C. James, Weakly compact sets, Trans. Amer. Math. Soc., 113 (1964), 129-140.

[11] L. Nachbin, Topology on Spaces of Holomorphic Mappings, Ergeb. der Math., 47, Springer-Verlag, Berlin, 1969.

[12] A. Pelczynski, On weakly compact polynomial operators on B-spaces with Dunford-Pettis property, Bull. de l'Acad. Polon. des Sciences, Sér. Sci. Math., 11 (1963), 371-378.

[13] R. A. Ryan, Dunford-Pettis properties, Bull. de l'Acad. Polon. des Sciences, Sér. Sci. Math., 27 (1979), 373-379.

Received September 12, 1986.

University College Galway

GALWAY, IRELAND

AND

Kent State UnIVERSITY

KENT, OH 44242 


\section{PACIFIC JOURNAL OF MATHEMATICS EDITORS}

\author{
V. S. VARADARAJAN \\ (Managing Editor) \\ University of California \\ Los Angeles, CA 90024 \\ Herbert Clemens \\ University of Utah \\ Salt Lake City, UT 84112 \\ R. FINN \\ Stanford University \\ Stanford, CA 94305
}

ROBION KIRBY

University of California

Berkeley, CA 94720

C. C. MOORE

University of California

Berkeley, CA 94720

HAROLD STARK

University of California, San Diego

La Jolla, CA 92093

\section{ASSOCIATE EDITORS}
R. ARENS
E. F. BECKENBACH
B. H. NEUMANN
F. WOLF
K. YOSHIDA (1906-1982)

\section{SUPPORTING INSTITUTIONS}
UNIVERSITY OF ARIZONA
UNIVERSITY OF OREGON
UNIVERSITY OF BRITISH COLUMBIA UNIVERSITY OF SOUTHERN CALIFORNIA
CALIFORNIA INSTITUTE OF TECHNOLOGY
UNIVERSITY OF CALIFORNIA
STANFORD UNIVERSITY
MONTANA STATE UNIVERSITY
UNIVERSITY OF HAWAII
UNIVERSITY OF NEVADA, RENO
UNIVERSITY OF TOKYO
NEW MEXICO STATE UNIVERSITY
UNIVERSITY OF UTAH
OREGON STATE UNIVERSITY
WASHINGTON STATE UNIVERSITY UNIVERSITY OF WASHINGTON 


\section{Pacific Journal of Mathematics}

\section{Vol. 131, No. $1 \quad$ November, 1988}

Tomek Bartoszynski, On covering of real line by null sets $\ldots \ldots \ldots \ldots \ldots 1$

Allen Davis Bell and Kenneth R. Goodearl, Uniform rank over differential operator rings and Poincaré-Birkhoff-Witt extensions $\ldots \ldots \ldots \ldots \ldots 13$

Brian Boe, Thomas Jones Enright and Brad Shelton, Determination of the intertwining operators for holomorphically induced representations of Hermitian symmetric pairs

Robert F. Brown, Topological identification of multiple solutions to

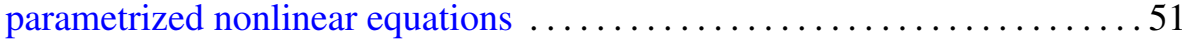

Marc R. M. Coppens, Weierstrass points with two prescribed nongaps ......71

Peter Larkin Duren and M. Schiffer, Grunsky inequalities for univalent functions with prescribed Hayman index .................... 105

Robert Greene and Hung-Hsi Wu, Lipschitz convergence of Riemannian manifolds

Kathryn E. Hare, Arithmetic properties of thin sets

Neal I. Koblitz, Primality of the number of points on an elliptic curve over a

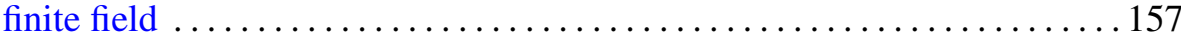

Isabel Dotti de Miatello and Roberto Jorge Miatello, Transitive isometry groups with noncompact isotropy

Raymond A Ryan, Weakly compact holomorphic mappings on Banach

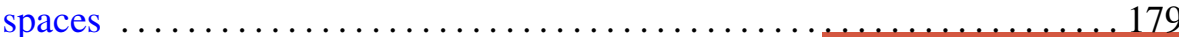

Tudor Zamfirescu, Curvature properties of typical convex surfaces 